Opioid analgesics are usually effective in the management of severe chronic pain. However, symptoms of opioid-induced bowel dysfunction (OIBD) are common during opioid therapy. Opioid-induced bowel dysfunction is often unsuccessfully managed due to limited effectiveness and numerous adverse effects of traditional laxatives. Newer treatment possibilities directed at the pathomechanism of OIBD comprise combined prolongedrelease oxycodone with prolongedrelease naloxone (oxycodone/naloxone) tablets. Oxycodone/naloxone provides effective analgesia with limited impact on bowel function as oxycodone displays high oral bioavailability and naloxone act as local antagonist on opioid receptors in the gastrointestinal tract due to nearly complete inactivation in the liver. Oxycodone/naloxone is administered to opioid-naive patients with severe pain and those unsuccessfully treated with weak opioids. Oxycodone/naloxone may be also administered to patients treated with strong opioids who experience intense symptoms of OIBD. Studies conducted to date indicate that oxycodone/naloxone is an important drug in chronic pain management, prevention and treatment of OIBD.

Key words: chronic pain, naloxone, opioid-induced bowel dysfunction, oxycodone, oxycodone/naloxone, pain treatment.

\section{The place of oxycodone/naloxone in chronic pain management}

\author{
Wojciech Leppert
}

Chair and Department of Palliative Medicine, Poznan University of Medical Sciences, Poznan, Poland

\section{Introduction}

Chronic pain treatment is based on the analgesic ladder established in 1986 by the World Health Organization (WHO) [1]. In most patients with cancer, pain is successfully relieved using opioids alone or in combination with adjuvant analgesics (co-analgesics) in accordance with the WHO analgesic ladder. Polish guidelines for the management of pain in cancer patients were updated [2]. Morphine and oxycodone (hydromorphone is currently not available in Poland), administered orally are recommended by the European Association for Palliative Care (EAPC) as the first choice opioids at the $3^{\text {rd }}$ step of the WHO analgesic ladder, which also comprises transdermal formulations of fentanyl and buprenorphine, methadone, and tapentadol (currently not available in Poland) for the treatment of moderate to severe chronic pain. In patients with mild to moderate pain two strategies are possible: either weak opioids or low doses of strong opioids: morphine up to $30 \mathrm{mg}$ or oxycodone up to $20 \mathrm{mg}$ per day (doses refer to the oral route) may be administered [3]. Opioids are usually accompanied with adjuvant analgesics to enhance analgesia (e.g. bisphosphonates in bone pain, anticonvulsants, antidepressants, local anesthetics, and NMDA receptor antagonists in neuropathic pain) [4, 5]. Supportive drugs such as laxatives and antiemetics may prevent or decrease opioid adverse effects [6]. Non-pharmacological measures, such as radiotherapy and invasive procedures (nerve blockades and neurolytic blocks) should be considered along with pharmacological measures to improve the effectiveness of pain therapy [7].

Opioids influence gastrointestinal (GI) tract function with numerous symptoms called opioid-induced bowel dysfunction (OIBD). Opioid-induced bowel dysfunction reflects a complex impact of opioids on the GI tract and comprise not only opioid-induced constipation (OIC), but also dry mouth, gastro-esophageal reflux-related symptoms (heartburn), nausea, vomiting, chronic abdominal pain, bloating, constipation-related symptoms: straining, hard stools, painful, infrequent and incomplete bowel movements, and diarrhea-related symptoms: urgency, loose bowel movements and frequent bowel movements [8]. The complex assessment of patients with symptoms of OIBD is necessary for the application of effective treatment [9].

Traditional laxatives are prescribed for the prevention and treatment of OIBD, particularly for OIC. However, they possess limited efficacy and display numerous adverse effects [10]. Moreover, treatment with laxatives should be limited to a short period of time. Still, in patients with chronic non-malignant and pain in the course of cancer, they usually have to be taken for the long-term as tolerance to the constipating effect of opioids does not develop [11]. Another approach is opioid switch or change from oral to transdermal or parenteral route of opioid administration. However, all opioids display OIBD including transdermal formulations [12].

Several newer strategies for the management of OIBD have been developed. They include administration of peripherally acting opioid receptor antag- 
onists (methylnaltrexone) and a combination of opioid analgesics with opioid antagonists. Methylnaltrexone is administered in the form of subcutaneous injections when treatment of OIC with traditional oral laxatives fails to provide bowel movements. This approach provides a bowel movement in approximately $50-60 \%$ of treated patients [13].

\section{Oxycodone/naloxone}

One of methods to decrease the frequency and severity of OIBD in patients requiring strong opioid administration due to moderate to severe pain is the use of an opioid analgesic with opioid receptor antagonist. The oral formulation of prolonged release (PR) oxycodone/PR naloxone (OXN) combines both drugs in one tablet [14]. The optimal $2: 1$ ratio of PR oxycodone/PR naloxone was demonstrated in a phase II study, rendering effective analgesia and improvement in bowel func tion with good treatment toleration in patients with severe chronic pain $[15,16]$. Oxycodone/PR naloxone is registered for the indication of severe pain, which may only be successfully treated with opioid analgesics; naloxone counteracts the development of OIBD through inhibition of oxycodone's effect on opioid receptors in the gut wall. The contraindications for OXN comprise bowel obstruction, acute abdominal conditions, diarrhea and allergy to the drug. Oxycodone/PR naloxone is available in several European countries including Poland. One pack contains 30 OXN tablets of $5 \mathrm{mg} / 2.5 \mathrm{mg}, 10 \mathrm{mg} /$ $5 \mathrm{mg}, 20 \mathrm{mg} / 10 \mathrm{mg}$, and $40 \mathrm{mg} / 20 \mathrm{mg}$ strength [17].

Following oral administration, oxycodone displays high bioavailability (60-87\%) and provides effective analgesia. The Acrocontin ${ }^{\circledR}$ system of PR oxycodone provides analgesia for $12 \mathrm{~h}$. An immediate analgesic effect is provided by $38 \%$ of the dose (plasma half-life of $37 \mathrm{~min}$ ) followed by a prolonged phase provided by $62 \%$ of the dose (plasma half-life of $6.2 \mathrm{~h}$ ). Oxycodone is metabolized mostly in the liver to inactive noroxycodone via CYP3A4 and to a lesser extent to active oxymorphone through CYP2D6; both noroxycodone and oxymorphone are subsequently converted to noroxymorphone via CYP2D6 and CYP3A4, respectively [18].

Naloxone undergoes extensive first-pass metabolism in the liver, with the formation of naloxone-3-glucuronide and displays low systemic bioavailability after oral administration $(<2 \%)$. The analgesic effect of oxycodone is not reversed by naloxone and no symptoms of opioid withdrawal are observed [19]. Naloxone binds to $\mu$-opioid receptors with a higher affinity than do opioid receptor agonists [20]. There is a clinically observed difference between immediate-release (IR) and PR formulations of naloxone. IR naloxone in some patients may attenuate analgesia or induce opioid withdrawal symptoms [21]. The PR naloxone formulation provides continuous occupation of gut opioid receptors and prevents the saturation of hepatic enzyme system responsible for naloxone metabolism and reduces the risk of opioid antagonism in the CNS [22]. The effect of orally administered naloxone depends on normal liver function, so any hepatic impairment should be carefully considered; in patients suffering from liver failure, OXN is not recommended [23]. A single and multiple doses pharmacokinetic study conducted among adult healthy subjects demonstrated bioequivalence of the combined OXN formulation and PR oxycodone and PR naloxone administered alone. It also demonstrated lack of interference between PR oxycodone and PR naloxone in a combined formulation indicating that the bioavailability of either compound is not affected [24]. In a controlled study conducted among healthy volunteers single dose of OXN 20 mg/10 mg significantly reduced mean colonic transit time (by $2.1 \mathrm{~h} ; p=0.0376$ ) compared to PR oxycodone $20 \mathrm{mg}$ dose administered alone [25].

The starting OXN dose in opioid-naive patients equals $5 \mathrm{mg} / 2.5 \mathrm{mg}-10 \mathrm{mg} / 5 \mathrm{mg}$ b.i.d. In patients not responding to weak opioids (tramadol, codeine, dihydrocodeine) initial doses of $10 \mathrm{mg} / 5 \mathrm{mg}$ or $20 \mathrm{mg} / 10 \mathrm{mg}$ b.i.d. are normally administered. When rotating from strong opioids to OXN starting doses are established individually, depending on the dose of previously administered opioid, analgesia, adverse effects, and co-morbidities. The maximal daily dose of OXN currently recommended is $80 \mathrm{mg} / 40 \mathrm{mg}$ daily [14]. However, higher daily doses up to $120 \mathrm{mg} / 60 \mathrm{mg}$ have been explored. In case of renal impairment the OXN dose should be reduced; in patients with renal failure other opioids such as buprenorphine and fentanyl may be considered. After OXN treatment completion and switch to another opioid the bowel function may deteriorate [26].

Adverse effects of OXN are generally similar to PR oxycodone; the frequency of diarrhea is slightly higher in OXN compared to PR oxycodone administered alone (5.2\% vs. 2.6\%) [27]. Oxycodone/PR naloxone less frequently induces nausea (6.3\% vs. $10.5 \%)$, vomiting (1.3\% vs. $4.3 \%)$, abdominal pain (1.3\% vs. $4.3 \%$ ) and dyspepsia (0.6\% vs. $2.5 \%$ ) in comparison to PR oxycodone administered alone [28]. These differences might be explained by naloxone's antagonist effect on gastric and gut opioid receptors [29] and in consequence naloxone prokinetic properties [30].

\section{Oxycodone/naloxone clinical studies}

\section{Patients with chronic non-malignant pain}

\section{Controlled studies}

Meissner et al. [15] reported a randomized, double-blind study that assessed analgesic efficacy and impact on the OIC of OXN, and identified the optimal dose ratio of oxycodone and naloxone. A total of 202 patients with chronic pain (most non-malignant, $2.5 \%$ cancer-related pain) and a stable oxycodone dose (40, 60 or $80 \mathrm{mg}$ per day) were randomized into groups that received 10,20 , and $40 \mathrm{mg}$ of naloxone daily or placebo. After 4 weeks of the maintenance phase, patients received PR oxycodone for 2 weeks. Pain intensity was evaluated by the NRS, and bowel function was assessed by the Bowel Function Index (BFI). No loss of analgesia with PR naloxone was observed. Bowel function improved with increasing PR naloxone dose. At pre-randomization, average scores of 48.0, 52.8, 49.4 and 46.2 were observed for placebo, in the $10 \mathrm{mg}, 20 \mathrm{mg}$ and $40 \mathrm{mg}$ PR naloxone groups, respectively, and at the end of maintenance the equivalent scores were 45.4, 40.3, 31.3 and 26.1 ( $p<0.05$ for $20 \mathrm{mg}$ and $40 \mathrm{mg}$ PR naloxone vs. placebo). In a quadratic response surface model with PR naloxone and PR oxycodone doses as factors, an improvement was observed with a decreasing oxycodone/naloxone ratio and appeared to plateau at the $2: 1$ ratio, with the overall effect at $2: 1$ approximately $50 \%$ greater 
than at $4: 1$. No loss of analgesic efficacy with naloxone was observed. Addition of up to $40 \mathrm{mg}$ of oral PR naloxone significantly reduced OIBD in patients with severe chronic pain who were established on PR oxycodone. Naloxone at doses of 20 and $40 \mathrm{mg}$ improved bowel function in comparison to the placebo $(p<0.05)$. The combination was well tolerated with no unexpected adverse effects. A trend towards an increase in diarrhea with the higher naloxone doses was observed. The $2: 1$ oxycodone/naloxone ratio was identified as the most suitable.

Nadstawek et al. [16] evaluated patient assessment of the efficacy and tolerability of oral PR oxycodone when co-administered with oral PR naloxone. A total of 202 patients with cancer and non-cancer pain who were on a stable PR oxycodone dose (40, 60 or $80 \mathrm{mg}$ per day) were randomized into groups that received 10, 20, $40 \mathrm{mg}$ of PR naloxone or placebo. After 4 weeks of the maintenance phase, patients were switched to PR oxycodone for 2 weeks. Efficacy was good or very good in $50 \%, 67.4 \%$ and $72.5 \%$ of patients in the 10 , 20 and $40 \mathrm{mg}$ PR naloxone group, respectively, compared to $43.5 \%$ of patients in the placebo group. Patient assessment of tolerability was ranked as good or very good by $83.3 \%$, $79.1 \%$ and $82.5 \%$ of patients in the 10,20 and $40 \mathrm{mg}$ per day PR naloxone dose group, respectively, compared with $71.7 \%$ of patients in the placebo group. The maintenance phase was preferred by patients in PR naloxone groups. Efficacy of a $2: 1$ dose ratio of oxycodone to naloxone was evaluated as good or very good by $70.4 \%$ of patients compared with $43.5 \%$ treated with placebo. Tolerability of the 2:1 dose ratio was ranked as good or very good by $81.5 \%$ of patients compared with $71.1 \%$ for the placebo group and patients preferred the maintenance phase.

Löwenstein et al. [19] in a randomized, double-blind, double dummy, parallel-group, and multicenter study assessed the use of higher OXN doses (converted from PR oxycodone 60-80 mg per day and allowed titrate the dose up to 120 $\mathrm{mg} /$ day) in patients with moderate-to-severe non-malignant pain and OIC. During the pre-randomization period, a total of 265 patients receiving opioids for moderate-to-severe nonmalignant pain were converted to PR oxycodone and titrated to an effective analgesic dose. Subsequently, patients were randomized to be treated either with OXN or PR oxycodone alone. A significant improvement in bowel function assessed by BFI ( $p<0.0001)$ after one week, an increase in complete spontaneous bowel movements (CSBM) per week (median 3.0 vs. 1.0) after 4 weeks of the treatment and lower laxative intake during the study period were observed in the OXN group compared to the PR oxycodone group. Pain intensity scores were comparable between the groups and consistent for the duration of the study. No unexpected adverse effects attributable to OXN were observed. The treatment with OXN was superior to PR oxycodone administered alone in terms of bowel function, while providing equivalent analgesia.

Vondrackova et al. [27] in a randomized, double-blind, placebo- and active-controlled, and parallel-group study demonstrated the superiority of OXN combination over placebo with respect to analgesic efficacy in patients with moderate to severe chronic low back pain. The full analysis population consisted of 463 patients. The times to recurrent pain events were significantly longer in the OXN group compared with placebo ( $p<0.0001-0.0003)$. Oxycodone/PR naloxone reduced the risk of pain events by $42 \%(p<0.0001)$. The occurrence of pain events was comparable for OXN vs. PR oxycodone, which confirmed that the addition of PR naloxone to PR oxycodone did not negatively influenced oxycodone analgesia. Oxycodone/PR naloxone provides patients with effective analgesia and improves OIBD. The safety profile of OXN is comparable to other opioids with the exception of OIC, which indicates that the addition of PR naloxone improves tolerability.

Simpson et al. [28] conducted a double-blind, multicenter trial in 322 adult patients with moderate-to-severe, noncancer pain who required opioids in a range of 20-50 mg per day of oxycodone. Patients were randomized to receive OXN or PR oxycodone for 12 weeks. The primary outcome was an improvement in constipation (BFI). The secondary assessments focused on pain intensity and additional bowel parameters. A significant improvement in BFI scores occurred with OXN compared with PR oxycodone after 4 weeks of the double-blind treatment ( -26.9 vs. -9.4 , respectively; $p<0.0001$ ), which was observed after only one week of the treatment and continued until study end. A significant increase in the number of CSBM and decrease in laxative use were also observed. The oxycodone analgesic efficacy was not compromised as pain intensity remained stable throughout the study period. The incidence of adverse events was comparable in both groups and typical for opioid analgesics. The fixed-ratio combination of OXN is superior to PR oxycodone alone and offers effective analgesia with significant improvement of OIC.

\section{Observational studies}

Sandner-Kiesling et al. [31] conducted long-term analysis over a period of up to 52 weeks of OXN therapy of the patients with chronic pain who had completed 12 weeks period in the above two phase III studies [27, 28]. The initial Brief Pain Inventory - Short Form (BPI-SF) scores $(3.9 \pm 1.52)$ of the average pain over the last 24 hours remained low after 6 months $(3.7 \pm 1.59)$ and 12 months $(3.8 \pm 1.72)$ of the treatment. The BPI-SF mean scores of pain interference with activity and with sleep remained low throughout the study period. The mean BFI score decreased from the initial $35.6 \pm 27.74$ to 20.6 \pm 24.01 after 12 months of the treatment. The treatment was well tolerated with typical opioid adverse effects. This study demonstrated that the treatment with OXN in daily doses of up to $80 \mathrm{mg} / 40 \mathrm{mg}$ was safe and effective.

Schutter et al. [32] assessed OXN efficacy and safety in a daily clinical practice. A total of 7836 patients from 6496 centers were included and followed for 4 weeks. The majority of them were opioid pre-treated (74.6\%), while $25.1 \%$ were opioid-naive. The pain causing underlying disease was musculoskeletal (85.9\%), and malignant disease (17.3\%) with multiple causes possible. The mean observation period was $33.6 \pm 13.2$ days. More than two-thirds of patients (68.3\%) started the treatment with OXN at a dose of $10 \mathrm{mg} /$ $5 \mathrm{mg}$ twice a day (minimum prescribed daily dose, $10 \mathrm{mg} / 5 \mathrm{mg}$; maximum prescribed daily dose, $60 \mathrm{mg} / 30 \mathrm{mg}$ ).

Analgesia (BPI-SF) improved; the strongest pain during the $24 \mathrm{~h}$ period prior to the first visit was $6.8 \pm 1.8$ and fell to 
$3.9 \pm 2.1$ at the final follow-up visit - a reduction of 2.9 points (95\% Cl: 2.8-3.0; $p<0.001$ ). Similar results were obtained for the least pain intensity, pain on average and pain right now ( $p<0.001$ for all differences). With respect to bowel function, it improved as the mean global BFI decreased from 38.2 \pm 30.7 at the first visit to $15.1 \pm 18$.5 at the third follow-up visit; it fell for both opioid-naive and opioid-pretreated patients, but as expected, the difference was more pronounced in the latter group.

Other symptoms frequency and severity improved comparing the first visit and the third follow-up visit: nausea, vomiting, constipation, diarrhea, abdominal pain, dizziness, and decreased appetite. Laxatives were taken by $28.2 \%$ (5.5\% of opioid-naive and $35.9 \%$ of opioid-pretreated patients) of patients at the first visit; at the final visit, $12.4 \%$ (3\% of opioid-naive and $18 \%$ of opioid-pretreated patients) took laxatives. Quality of life (QOL) improved by $43 \%$. The most frequent adverse effects were nausea and constipation (3.8\% of patients each) and dizziness (2.8\%). A total of 244 serious adverse events (SAE) were documented in 177 patients (2.3\%). In the majority of patients with SAE there was no causal relationship with the use of OXN in the opinion of the treating physicians and the sponsor.

As part of this study [32], Hermanns et al. [33] conducted an analysis of OXN (in the daily dose range of $20 \mathrm{mg} / 10$ $\mathrm{mg}$ to $40 \mathrm{mg} / 20 \mathrm{mg}$ ) administered over a period of up to 4 weeks in 1488 patients with severe neuropathic non-malignant pain. During the treatment with $\mathrm{OXN}$, the mean pain intensity decreased in both opioid-naive and opioid-pretreated patients. After 4 weeks of the treatment, the BFI scores dropped from the initial $41.6 \pm 31.6$ to $16.5 \pm 19.6$ ( $p<0.001$ ), reflecting normal bowel function. Additionally, QOL was improved by $47 \%$. Oxycodone/PR naloxone provided effective analgesia and improved bowel function measured by BFI and a significant improvement in quality of life over the period of 4 weeks of the treatment.

Gatti et al. [34] in a retrospective, single center, observational study assessed the effectiveness and safety of OXN in consecutive patients with constipation and chronic nonmalignant pain, both in opioid-naive and opioid-tolerant patients. Efficacy was assessed by pain intensity, bowel function, effective OXN dose, the Patient Global Impression of Change (PGIC) scale, rescue paracetamol, and laxative use. Of 1,051 patients starting on the OXN, 1,012 completed 2 months treatment. Oxycodone/PR naloxone significantly decreased pain intensity $(p<0.001)$, reduced need for rescue paracetamol ( $p<0.001)$; PGIC score "very much improved" or "much improved" in $84.0 \%$ patients. Constipation markedly decreased $(p<0.001)$ despite a reduced laxative use ( $p<0.001$ vs. baseline). The most frequent adverse events were somnolence (2.0\%), dizziness (1.1\%), and confusion (1.0\%). Clinical differences in endpoints were observed between opioid-naive and opioid-tolerant patients, and among age stratified groups, but efficacy was similar to that of the overall population. Oxycodone/PR naloxone was effective and well tolerated in moderate-to-severe chronic pain in patients with constipation, providing analgesia and relief from bowel dysfunction. Consistent efficacy across patient subgroups provides guidance for daily management of chronic pain when therapy options are limited due to bowel dysfunction, regardless of age or previous medication.

Dunlop et al. [35] compared cost-effectiveness and QOL of OXN and PR oxycodone in patients with moderate-tosevere non-malignant pain and OIC. Although the PR oxycodone/PR naloxone direct treatment cost was slightly higher than that of oxycodone PR, when analyzing constipation treatment costs and benefits of OXN in terms of improved quality-adjusted life-year, PR oxycodone/PR naloxone was the cost-effective option in UK.

\section{Patients with cancer pain}

Ahmedzai et al. [36] in a randomized, double-blind, multicentre study, assessed OXN analgesic efficacy, tolerance of the treatment, and impact on constipation, and compared it to PR oxycodone in patients with moderate-to-severe cancer pain. A total of 185 patients were randomized to receive up to $120 \mathrm{mg} / 60 \mathrm{mg}$ per day of OXN or $120 \mathrm{mg}$ PR oxycodone over 4 weeks. After 4 weeks, mean BFI and Patient Assessment of Constipation Symptoms (PAC-SYM) scores were significantly lower, with OXN and the mean total laxative intake was 20\% lower in this patient group than in the case of PR oxycodone. The mean BPI-SF scores were similar for both treatments and the consumption of rescue analgesics was low and comparable between the two patient groups. QOL (assessed by the EORTC QLQ-C30 and the EuroQoL) results showed better scores with respect to constipation-related symptoms in the group treated with OXN. Adverse effects were similar in both patient groups. Specifically, no difference in scores of the modified Subjective Opiate Withdrawal Scale (SOWS) was found between patient groups. The results suggest that OXN in doses of up to $120 \mathrm{mg} / 60 \mathrm{mg}$ per day may provide effective analgesia and improve bowel function.

Clemens et al. [37] conducted an uncontrolled study among 26 patients with advanced cancer who received different opioids due to severe pain. The former opioid treatment was switched to OXN at a maximum daily dose of $40 \mathrm{mg} / 20 \mathrm{mg}$ that was administered for a period of 14 days. Bowel function was assessed by BFI, the Bristol Stool Form Scale (BSFS) and the PGIC. In 21 patients, constipation improved, as measured by BFI, BSFS, and PGIC, while providing adequate analgesia. The most frequent adverse effects were nausea (9 patients) and abdominal pain (5 patients). Two patients experienced diarrhea. Opioid withdrawal symptoms were not observed. In 5 patients it was necessary to switch to hydromorphone due to inadequate pain relief.

In a case report, Mercadante [38] depicted a cancer patient with severe pain who required high OXN daily doses $(240 \mathrm{mg}$ / $120 \mathrm{mg}$ ) which were ineffective. A switch to PR oxycodone alone at a daily dose of $240 \mathrm{mg}$ provided satisfactory analgesia. This may suggest that at higher doses (240 mg/120 mg per day) OXN provides inferior analgesia compared to PR oxycodone administered alone at a dose of $240 \mathrm{mg}$ daily.

\section{The place of oxycodone/naloxone in the management of chronic pain and opioid-induced bowel dysfunction}

Oxycodone/PR naloxone provides similar analgesia to PR oxycodone, with improvement in bowel function as docu- 
2

Drug(s) of the step I (oral):

- Osmotic agents: lactulose or macrogol

- Stimulants: anthranoids or polyphenolics

- Oxycodone/naloxone
Invasive procedures of the step III:

- Rectal enema

- Manual evacuation**

Drug(s) of the step II:

- Rectal suppositories

- PAMORA*

(Methylnaltrexone - s.c.)

Constipation persists or intensifies

1

OXN should be considered in patients who require opioid administration for pain and who are at high risk of OIBD development.

*PAMORA - peripherally acting mu-opioid receptor antagonists (methylnaltrexone administered by the subcutaneous route) indicated for patients who do not respond to traditional oral laxatives without bowel obstruction and acute abdominal illness [43].

**This procedure should be used only when other measures fail and the faecal impaction causes significant pain and distress for the patient. It should be preceded by administration of sedative and analgesics (local and systemic) that provide effective relief of severe pain and distress associated with manual stool evacuation.

Fig. 1. The three-step ladder of the treatment of opioid-induced constipation [40]

mented by better results of BFI, PAC-SYM, more frequent CSBMs and lower consumption of laxatives comparing to PR oxycodone. Oxycodone/PR naloxone renders similar analgesia and reverses OIC in comparison to PR oxycodone administered alone in patients with chronic non-malignant pain $[27,28]$, cancer patients with pain [36], and in patients with postoperative pain [39], thus it improves patients' compliance and QOL. Currently the drug is registered in daily doses up to $80 \mathrm{mg} / 40 \mathrm{mg}$. However, a controlled study conducted in cancer patients demonstrated daily doses $120 \mathrm{mg} / 60 \mathrm{mg}$ may be safe and effective [36]. Oxycodone/PR naloxone may be administered to opioid-naive patients with moderateto-severe pain, to patients not responding to weak opioids and to patients rotated from other opioids, which cause severe symptoms of OIBD.

The Expert Working Group of the Polish Society of Palliative Medicine developed a three step ladder for the management of OIC (Fig. 1) [40]. Studies conducted in patients with chronic non-malignant and cancer pain support the important role of OXN in prevention and treatment of OIC in patients who require opioids for moderate-to-severe pain, therefore OXN consideration at the first step of the ladder of OIC treatment is suggested. Recent recommendations of the European Society for Clinical Oncology for pain management in cancer patients [41] and UK experts' for the management of myeloma patients' [42] suggests the use of OXN.

Oxycodone/PR naloxone plays an important role in patients with OIBD as traditional laxatives are often ineffective. Oxycodone/PR naloxone targets the source of OIBD as naloxone blocks opioid receptors in the gut and oxycodone provides effective analgesia. These targeted mechanisms of OXN action have the potential advantage as the drug may not only treat but also prevent OIBD development. Thus, OXN administration may be considered in cancer pain patients who are at high risk of OIBD development such as those with Gl tumors, patients who require combined treatment with opioids and other drugs disturbing normal bowel function. This especially applies to high-risk populations, such as advanced cancer patients who usually have several factors disturbing normal bowel function. Future clinical studies may address OXN analgesic efficacy, safety, cost-effectiveness, and patients' QOL.

The author declares no conflict of interest.

\section{References}

1. World Health Organisation: Cancer Pain Relief and Palliative Care. Geneva: World Health Organisation, 1996.

2. Krajnik M, Wordliczek J, Dobrogowski J. Standards of cancer pain treatment - state of the art in 2012 (in Polish). Terapia 2012; 20: 6-13.

3. Caraceni A, Hanks G, Kaasa S, et al. for the European Palliative Care Research Collaborative (EPCRC) on behalf of the European Association for Palliative Care (EAPC): Use of opioid analgesics in the treatment of cancer pain: evidence-based recommendations from the EAPC. Lancet Oncol 2012; 13: e58-e68.

4. Hanks GW, de Conno F, Cherny N, et al. Expert Working Group of the Research Network of the European Association for Palliative Care: Morphine and alternative opioids in cancer pain: the EAPC recommendations. Br J Cancer 2001; 84: 587-93.

5. Lussier D, Huskey AG, Portenoy RK. Adjuvant Analgesics in Cancer Pain Management. Oncologist 2004; 9: 571-91.

6. Leppert W. Pain Management in patients with cancer: focus on opioid analgesics. Curr Pain Headache Rep 2011; 15; 271-9.

7. Eidelman A, White T, Swarm RA. Interventional therapies for cancer pain management: important adjuvants to systemic analgesics. J Natl Compr Canc Netw 2007; 5: 753-60.

8. Brock C, Olesen SS, Olesen AE, Frøkjaer JB, Andresen T, Drewes AM. Opioid-Induced Bowel Dysfunction. Pathophysiology and Management. Drugs 2012; 72: 1847-65.

9. Rentz AM, Yu R, Müller-Lissner S, Leyendecker P. Validation of the Bowel Function Index to detect clinically meaningful changes in opioid-induced constipation. J Med Econ 2009; 12: 371-83.

10. Larkin PJ, Sykes NP, Centeno C, et al. The management of constipation in palliative care: clinical practice recommendations. Palliat Med 2008; 22: 796-807.

11. Holzer P, Ahmedzai SH, Niederle N, et al. Opioid-induced bowel dysfunction in cancer-related pain: causes, consequences, and a novel approach for its management. J Opioid Manage 2009; 5: 145-95. 
12. Leppert $\mathbf{W}$. The impact of opioid analgesics on the gastrointestinal tract function and the current management possibilities. Wspolczesna Onkol 2012; 16: 125-31.

13. Bader S, Dürk T, Becker G. Methylnaltrexone for the treatment of opioid-induced constipation. Expert Rev Gestroenterol Hepatol 2013; 7 : 13-26.

14. Leppert W. Role of oxycodone and oxycodone/naloxone in cancer pain management. Pharmacol Rep 2010; 62: 578-91.

15. Meissner W, Leyendecker P, Mueller-Lissner S, et al. A randomised controlled trial with prolonged-release oral oxycodone and nalox one to prevent and reverse opioid-induced constipation. Eur J Pain 2009; 13: 56-64.

16. Nadstawek J, Leyendecker P, Hopp M, Ruckers C, Wirz S, Fleischer W, Reimer K. Patient assessment of a novel therapeutic approach for the treatment of severe, chronic pain. Int J Clin Pract 2008; 62: 1159-67.

17. Anonymous. Oxycodone/naloxone prolonged release tablets, ques tions and answers. Paineurope supplement, February 2009.

18. Riley J, Eisenberg E, Müller-Schwefe G, Drewers AM, Arendt-Nielsen $L$. Oxycodone: a review of its use in the management of pain. Curr Med Res Opin 2008; 24: 175-92.

19. Löwenstein O, Leyendecker P, Hopp M, et al. Combined prolonged release oxycodone and naloxone improves bowel function in patients receiving opioids for moderate-to-severe non-malignant chronic pain: a randomized controlled trial. Expert Opin Pharmacother 2009; 10: 531-43.

20. Clemens KE, Mikus G. Combined oral prolonged-release oxycodone and naloxone in opioid-induced bowel dysfunction: review of efficacy and safety data in the treatment of patients experiencing chronic pain. Expert Opin Pharmacother 2010; 11: 297-310.

21. Liu M, Wittbrodt E. Low-dose oral naloxone reverses opioid-induced constipation and analgesia. J Pain Symptom Manage 2002; 23 48-53.

22. Leppert $W$. The management of patients with gastrointestinal symptoms in palliative medicine (in Polish). Terapia 2011; 19: 59-66.

23. Reimer K, Hopp M, Zenz M, et al. Meeting the challenges of opioidinduced constipation in chronic pain management - a novel approach. Pharmacology 2009; 83: 10-7.

24. Smith K, Hopp M, Mundin G, Leyendecker P, Bailey P, Grothe B, Uhl R, Reimer K. Single- and multiple-dose pharmacokinetic evaluation of oxycodone and naloxone in an opioid agonist/antagonist prolonged-release combination in healthy adult volunteers. Clin Ther 2008; 30: 2051-68.

25. Smith K, Hopp M, Mundin G, et al. Naloxone as a part of a prolonged release oxycodone/naloxone combination reduces oxycodoneinduced slowing of gastrointestinal transit in healthy volunteers. Expert Opin Invest Drugs 2011; 20: 427-39.

26. Oxycodone/naloxone. Medical Product Characteristics (in Polish). Mundipharma Polska 16.08.2011.

27. Vondrackova D, Leyendecker P, Meissner W, et al. Analgesic efficacy and safety of oxycodone in combination with naloxone as prolonged release tablets in patients with moderate to severe chronic pain. J Pain 2008; 9: 1144-54.

28. Simpson K, Leyendecker P, Hopp M, et al. Fixed-ratio combination oxycodone/naloxone compared with oxycodone alone for the relief of opioid-induced constipation in moderate-to-severe noncancer pain. Curr Med Res Opin 2008; 24: 3503-12

29. Smith K, Hopp M, Mundin G, Bond S, Bailey P, Woodward J, Bell D. Low absolute bioavailability of oral naloxone in healthy subjects. Int J Clin Pharmacol Ther 2012; 50: 360-7.

30. Schang JC, Devroede G. Beneficial effects of naloxone in a patient with intestinal pseudoobstruction. Am J Gastroenterol 1985; 80: 407-11.

31. Sandner-Kiesling A, Leyendecker P, Hopp M, et al. Long-term efficacy and safety of combined prolonged-release oxycodone and naloxone in the management of non-cancer chronic pain. Int J Clin Pract 2010; 64: 763-74.

32. Schutter U, Grunert S, Meyer C, Schmidt T, Nolte T. Innovative pain therapy with a fixed combination of prolonged-release oxycodone/naloxone: a large observational study under conditions of daily practice. Curr Med Res Opin 2010; 26: 1377-87.
33. Hermanns K, Junker U, Nolte T. Prolonged-release oxycodone/naloxone in the treatment of neuropathic pain - results from a large observational study. Exp Opin Pharmacother 2012; 13: 299-311.

34. Gatti A, Casali M, Lazzari M, Tufaro G, Gafforio P, Silvestri C, Marcassa C, Sabato AF. Prolonged-release oxycodone/naloxone in nonmalignant pain: single-center study in patients with constipation. Adv Ther 2013; 30: 41-59.

35. Dunlop W, Uhl R, Khan I, Taylor A, Barton G. Quality of life benefits and cost impact of prolonged release oxycodone/naloxone versus prolonged release oxycodone in patients with moderate-to-severe non-malignant pain and opioid-induced constipation: a UK cost-utility analysis. J Med Econ 2012; 15: 564-75.

36. Ahmedzai SH, Nauck F, Bar-Sela G, Bosse B, Leyendecker P, Hopp $M$. A randomized, double-blind, active-controlled, double-dummy, parallel-group study to determine the safety and efficacy of oxycodone/naloxone prolonged-release tablets in patients with moderate/severe, chronic cancer pain. Palliat Med 2012; 26: 50-60.

37. Clemens KE, Quednau I, Klaschik E. Bowel function during pain therapy with oxycodone/naloxone prolonged-release tablets in patients with advanced cancer. Int J Clin Pract 2011; 65: 472-8.

38. Mercadante S, Ferrera P, Adile C. High doses of oxycodone-naloxone combination may provide poor analgesia. Support Care Cancer 2011; 19: 1471-72.

39. Kuusniemi K, Zöllner J, Sjövall S, et al. Prolonged-release oxycodone/naloxone in postoperative pain management: from a randomized clinical trial to usual clinical practice. J Int Med Res 2012; 40: 1775-93

40. Leppert W, Dzierzanowski T, Cialkowska-Rysz A, Jarosz J, Pyszkowska J, Stachowiak A. The management of constipation in palliative medicine - recommendations of the Expert Working Group of the Polish Association for Palliative Medicine (in Polish). Med Paliat 2009; 1: $1-8$.

41. Ripamonti Cl, Santini D, Maranzano E, Berti M, Roila F; ESMO Guidelines Working Group. Management of cancer pain: ESMO Clinical Practice Guidelines. Ann Oncol 2012 (supplement 7): vii139-54.

42. Snowden JA, Ahmedzai SH, Ashcroft J, et al.; Haemato-oncology Task Force of British Committee for Standards in Haematology and UK Myeloma Forum. Guidelines for supportive care in multiple myeloma 2011. Br J Haematol 2011; 154: 76-103.

43. Leppert $W$. The role of opioid receptor antagonists in the treatment of opioid-induced constipation - a review. Adv Ther 2010; 27: 714-30.

\section{Address for correspondence}

\section{Wojciech Leppert}

Chair and Department of Palliative Medicine

Poznan University of Medical Sciences

Osiedle Rusa 25 A

61-245 Poznan, Poland

tel. and fax: + 48618738303

e-mail: wojciechleppert@wp.pl

Submitted: 25.03 .2013

Accepted: 16.04 .2013 